\title{
Emotion recognition using electroencephalogram signal
}

\author{
Aqila Nur Nadira Mohammad Yosi ${ }^{1}$, Khairul Azami Sidek ${ }^{2}$, Hamwira Sakti Yaacob ${ }^{3}$, \\ Marini Othman ${ }^{4}$, Ahmad Zamani Jusoh ${ }^{5}$ \\ ${ }^{1,2,5}$ Department of Electrical and Computer Engineering, Kulliyyah of Engineering, \\ International Islamic University Malaysia, Malaysia \\ ${ }^{3}$ Department of Computer Science, Kulliyyah of Information and Communication Technology, \\ International Islamic University Malaysia, Malaysia \\ ${ }^{4}$ Department of Information System, Kulliyyah of Information and Communication Technology, \\ International Islamic University Malaysia
}

\begin{tabular}{l}
\hline \hline Article Info \\
\hline Article history: \\
Received Oct 28, 2018 \\
Revised Jan 23, 2019 \\
Accepted Mar 5, 2019
\end{tabular}

Keywords:

EEG

Electroencephalogram emotion

\begin{abstract}
Emotion play an essential role in human's life and it is not consciously controlled. Some of the emotion can be easily expressed by facial expressions, speech, behavior and gesture but some are not. This study investigates the emotion recognition using electroencephalogram (EEG) signal. Undoubtedly, EEG signals can detect human brain activity accurately with high resolution data acquisition device as compared to other biological signals. Changes in the human brain's electrical activity occur very quickly, thus a high resolution device is required to determine the emotion precisely. In this study, we will prove the strength and reliability of EEG signals as an emotion recognition mechanism for four different emotions which are happy, sad, fear and calm. Data of six different subjects were collected by using BrainMarker EXG device which consist of 19 channels. The pre-processing stage was performed using second order of low pass Butterworth filter to remove the unwanted signals. Then, two ranges of frequency bands were extracted from the signals which are alpha and beta. Finally, these samples will be classified using MLP Neural Network. Classification accuracy up to $91 \%$ is achieved and the average percentage of accuracy for calm, fear, happy and sad are $83.5 \%, 87.3 \%, 85.83 \%$ and $87.6 \%$ respectively. Thus, a proof of concept, this study has been capable of proposing a system of recognizing four states of emotion which are happy, sad, fear and calm by using EEG signal.
\end{abstract}

Copyright (C) 2019 Institute of Advanced Engineering and Science. All rights reserved.

\section{Corresponding Author:}

Aqila Nur Nadira Mohammad Yosi,

Department of Electrical and Computer Engineering,

International Islamic University Malaysia,

Jalan Gombak 53100, Kuala Lumpur, Malaysia.

Email: aqilanurnadira@gmail.com

\section{INTRODUCTION}

In psychology, emotion is a complex state of feelings that results in physical and psychological changes that influence thought and actions of an individual. Emotion can be considered as an experience of an individual due to interactions with the environment. Every day, human produce varieties of emotions and it can be related to many possibilities such as events, people, objects, self-appraisal and many more. Emotions play an essential role in human's life and it is not consciously controlled. Some of the emotion can be easily expressed by facial expressions, speech, behavior and gesture but some are not [1,2]. Controlling of emotions are important especially during decision making, communication and survival. For example, people tend to be lose concentration if they are too immersed in their emotion while driving on the road. 
Some of the possibilities that can affect emotions during driving are stress in a workplace, receiving upsetting news, involve in arguments or running late to a certain event.

Failure to express emotions also may lead a person to emotional upheaval. It can be defined as surge of emotion that causes major psychological distress for an individual. It is more likely to happen due to unexpected lifestyle changes such as divorce or nervous breakdown which can cause an individual to experience trauma and mental illness. There are a lot of factors that can cause an individual to have emotional upheaval such as mental illness like bipolar disorder, depression and anxiety. People with mental illness are more likely to have emotional upheaval of unpleasant emotion [3].

Thus, it is proven that identifying emotions of an individual in most of the situation is vital. A lot of researches have been performed in order to recognize emotions from speech, facial expressions or both [4-6]. However, these methods have limitations and challenges as not all emotions can be obviously expressed through facial expression, speech and actions. Outward expression can be controlled due to environment or some other circumstances [7-9].

Recently, biological signals such as photoplethysmogram (PPG), electroencephalogram (EEG), electrocardiogram (ECG) and acceleration plethysmogram (APG) have been proposed as the method to detect emotion variabilities. All this information can be obtained through physiological instruments that measure the heart rate, blood pressure, oxygen saturation levels, blood glucose, nerve conduction and brain activity. However, this study will be focus more on recognizing emotions by using EEG signals. This is because it can detect human brain activity accurately with high resolution data acquisition device as compared to other biological signals [10-12]. Changes in the human brain's electrical activity occur very quickly, thus a high-resolution device is required to determine the emotion precisely [13, 14]. EEG signals are measured through electrical brain waves by placing the scalp over multiple areas of the brain as shown in Figure 1.

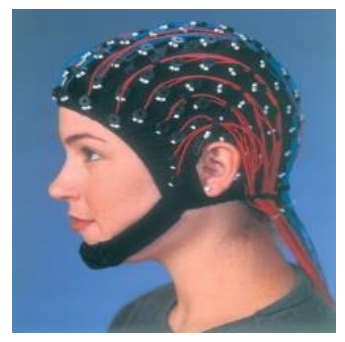

Figure 1. Recording user's brain wave using EEG [15]

There are many advantages of using EEG signals and one of them is that emotions cannot be freely controlled which makes it more accurate as compared to other methods such as facial expressions, speech etc. Furthermore, EEG signal is continuously available at all time, even though the person is asleep or awake [16]. Hence, this study proposed a system of recognizing four states of emotion which are calm, fear, happy and sad using EEG signal. The objectives of this study are; to study the EEG signals and its characteristics, to develop the EEG based recognition system as well as to assess the performance of the system.

The rest of this paper is organized as follows. Section 2 summarizes the previous works that are related to this study while Section 3 presents the research methodology by explaining the data acquisition, preprocessing, future extraction and classification of emotions processes. Further discussion and results will be further elaborated in Section 4. Finally, Section 5 concludes the study as well as mapped the future work.

\section{RELATED WORKS}

In recent years, there has been a growing interest from all researchers around the world in recognizing variability of human's emotion from facial expressions, speech and both. However, in this study, it is more focused on emotion recognition that uses electroencephalogram (EEG) signals as it is relatively a new method used by researchers. There are a lot of techniques that have been used in analyzing the EEG signals as well as to classify those signals into various types of emotions.

Horlings et al. in [17] access human emotion by using EEG signals. Two states that have been put into attention are valence and arousal. Some pictures are showed to the subjects in order to arouse emotions. It is found out that Support Vector Machine (SVM) performs the best in emotion classification where 32\% 
and $37 \%$ of classification rates for valence and arousal are achieved respectively. In addition, higher performance of classification rates was obtained by using only the extreme values where the rates for valence are $71 \%$ and arousal are $81 \%$. However, this system did not consider the variety among different people and conditions as new samples that were applied and gave only $35 \%$ correct accuracy rate.

Nie et al. in [18] classified two types of emotions which are positive and negative, by using EEG signals. Bandpass filter was applied in order to extract the EEG data to delta, theta, alpha, beta and gamma waves. Based on the result, alpha, beta and gamma bands showed higher performance in detecting emotions compared to delta and theta bands. This can be concluded that bands with higher frequency are essential in recognizing emotions. However, $87.53 \%$ of average accuracy rate was obtained which provided room of improvement as $12.47 \%$ error exists.

Soleymani et al. in [19] proposed a different approach in recognizing emotion by using EEG signals and facial expressions. The study used frontal view video in order to capture the facial expressions for each subject. Subjects were asked to watch 20 short videos from famous commercially produced movies to evoke different emotions. Unwanted artifacts and noise are filtered by preprocessing and a sampling rate of $256 \mathrm{~Hz}$ were used in this study. Long Short Term Memory Neural Networks technology was applied and in order to segment and classify data, Conditional Random Fields (CRF) was employed instead of Hidden Markov Models (HMM). Results showed electrodes positioned on the frontal, parietal and occipital lobes with high frequency obtained higher correlation which carries important information regardless of the contamination from facial muscular activities. However, the results will be affected if the subjects are not in stationary position which error can easily occur and delay can be experienced due to continuous annotation of facial expressions.

Although there are quite a lot of researches being made to differentiate the variability in human's emotion, including the EEG itself, there are still improvements that can be made in order to increase the accuracy of the results obtained. Thus, this study will propose an emotion recognition technique using EEG signal.

\section{RESULTS AND ANALYSIS}

Methodology is the analysis of specific procedures or methods used to analyze the information in order to obtain the result required. This chapter will briefly discuss about the proposed methodology used to study the recognition of emotion using EEG signal. In this study, calm, fear, happy and sad are the four emotions to be recognized. Four steps are used to detect and recognize emotion variabilities from individuals which are data acquisition, pre-processing, feature extraction and classification as shown in Figure 2.

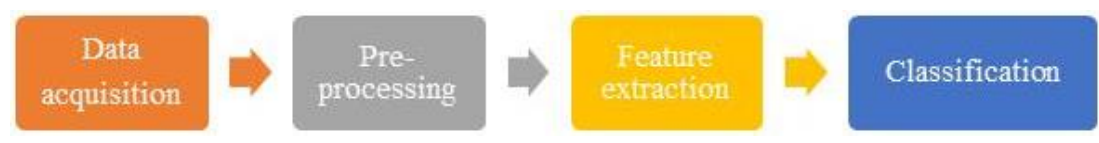

Figure 1. Method used for emotion recognition using EEG signal

\subsection{Data Acquisition}

Data acquisition is the first part of the process where the EEG signals are acquired from six different subjects by using an EEG device from Kulliyyah of Information Computer and Technology (KICT), named Brainmarker. The device is connected to 19 electrodes which represents the 19 channels of EEG signals as shown in Figure 3.

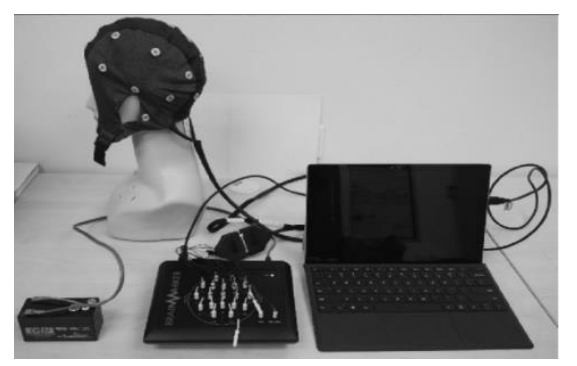

Figure 2. EEG device namely brainmarker EXG [20] 
All subjects are students from International Islamic University Malaysia (IIUM) with age range from 22 to 24 years old and in good health condition. These subjects need to watch four videos in order to stimulate calm, sad, fear and happy emotions. Before the videos are started, the subjects were given a headphone so that they will be more focused on the video without any external distraction. However, EEG signal is very sensitive to movements. Therefore, all subjects are advised not to make any movement and minimize eye blinking as it will give major effect to the EEG signal.

\subsection{Pre-processing}

After the EEG signal is acquired, these data signals need to go through pre-processing. Pre-processing is a state of process to remove noise from the signals. Since brain activity only produces very weak signals, EEG signals will contain a lot of background noise [21]. There are many sources of noise such as static electricity or electromagnetic fields produced by the nearby devices, artifacts and any other external influences. In this study, second order low pass Butterworth filter is used at the pre-processing stage as all the relevant information especially for emotion classification are found in the frequencies below $30 \mathrm{~Hz}$.

\subsection{Feature Extraction}

The second stage of this study is feature extraction where all the useful information from the signals will be extracted. Based on previous study, high frequency bands play an essential role in recognizing human emotion variabilities [18]. Therefore, in this part, EEG signals will be selected based on their frequency band which are alpha bands and beta bands. The range of frequencies for the bands are between (8-13) Hz and (13$30) \mathrm{Hz}$ respectively. In this study, wavelet transform is used in order to split the signals into selected frequency bands. Multi-resolution analysis of "db8" wavelet function is used for decomposing the EEG signals into five levels and two frequency bands which are alpha and beta.

\subsection{Classification}

After extracting the selected features, the classification of emotion will be done by suitable classifiers where this is the last stage of this study. Classification is the process of grouping the related data into a single group. Hence in this study, Multilayer Perceptron (MLP) is used. It is a feedforward artificial neural network model that plots sets of input data onto a set of outputs. All the network parameters adapted are shown in Table 1.

Table 1. Parameter of Neural Network

\begin{tabular}{cc}
\hline Parameters & Values \\
\hline Number of input layer & 12 \\
Number of hidden layer & 2 \\
Number of output layer & 2 \\
Number of epochs & 1000 \\
Mean square error & 0.1 \\
\hline
\end{tabular}

In this study, the result of classification process will be based on the Russell's Circumplex Model of Effect as it is one of the commonly used model of emotions as shown in Figure 4 [22-25]. Each emotion is placed according to their level of arousal and valence. Arousal ranges from excited to calm while valence ranges from pleasant to unpleasant.

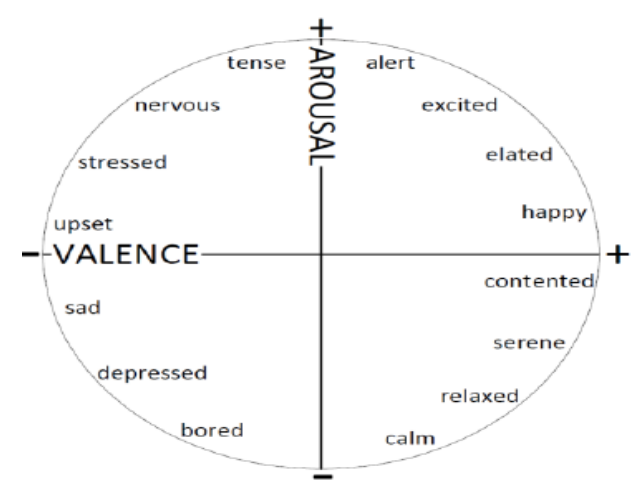

Figure 3. Russell's circumplex model of effect [24] 


\section{RESULTS AND ANALYSIS}

In this study, EEG data were taken from six different subjects age ranged from 22 to 24 years old and all of them were in good health condition. They are required to watch four different videos to stimulate calm, fear, happy and sad emotions. In order to acquire the EEG data, subjects were required to wear a cap that consist of 19 electrodes. The cap was connected to a device and a laptop. Brainmarker software are used for the data acquisition process. Based on previous research, some of the electrodes gave higher accuracy rate in classifying emotions [24].

Therefore, in this study, T8, F3, P3 and P4 channels were used to analyze happy, calm, fear and sad respectively. The number of samples in each signal is depends on the time duration of the video clips. Figure 5 and Figure 6 display the raw data of subjects 1 and 2 respectively for all emotion state.
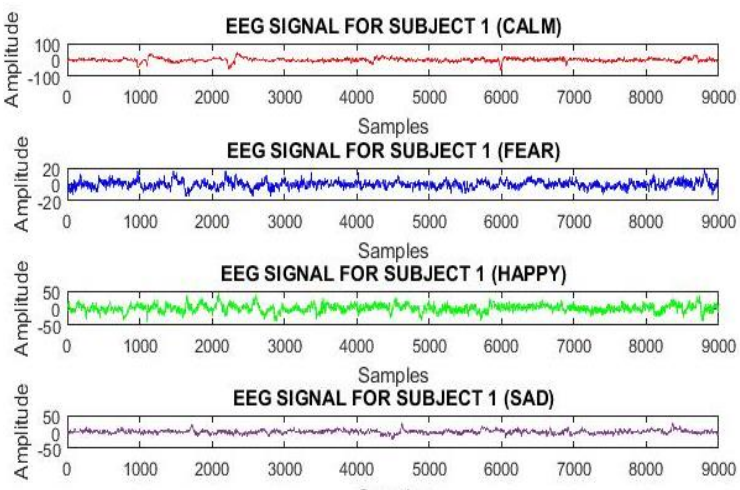

Samples
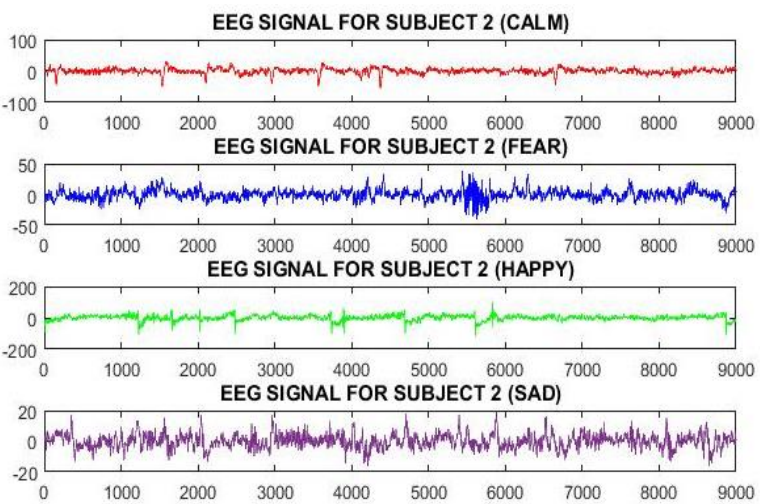

Figure 5. Raw EEG signal for Subject 2

Figure 4. Raw EEG signal for Subject 1

The outcome from the first process of this study which is data acquisition from 6 different subjects showed that different people have different waveforms for each emotion. The next stage after data acquisition is pre-processing. The noise or unwanted signal may be caused due to the movement of the subjects or the instability of the subjects during data acquisition process. Therefore, preprocessing is needed to get the smooth signal waveforms. In this study, second order low pass Butterworth filter with normalized cutoff frequency of $0.25 \mathrm{~Hz}$ is used. The filtered signal of subjects 1 and 2 are shown in Figure 7 and Figure 8 . The output showed smooth waveforms of EEG signals.

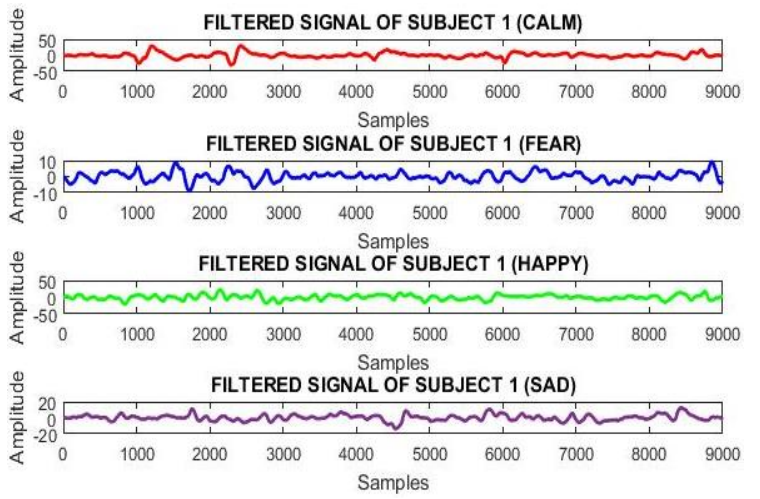

Figure 6. Filtered EEG signal for Subject 1

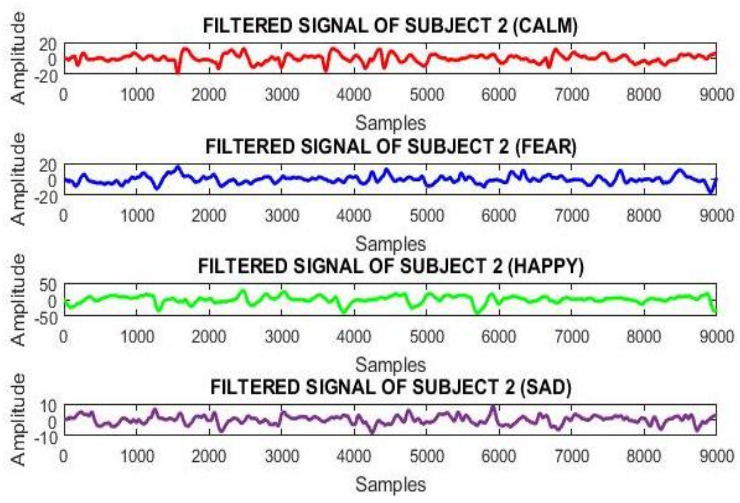

Figure 7. Filtered EEG signal for Subject 2

After undergoing the pre-processing stage, the signals are then extracted according to frequency bands. High frequency band which are alpha and beta, play an important role in emotion classification. Hence, signal with frequency (8-13) Hz and (13-30) Hz are extracted to represent alpha and beta respectively by using Wavelet Transform. Figures 9 until 16 show the alpha and beta signals of each emotion for subjects 1 and 2. 

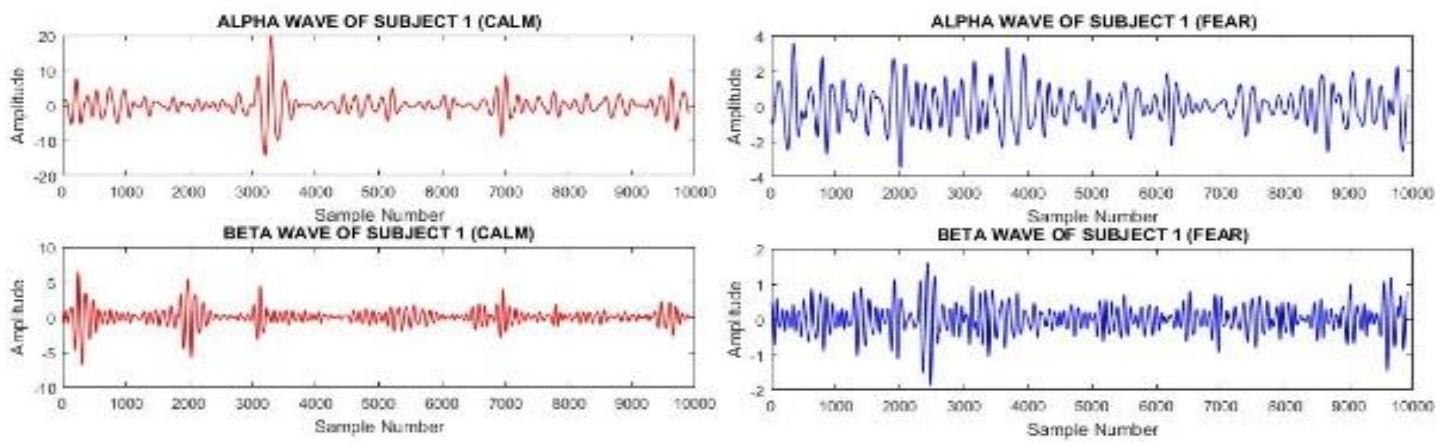

Figure 9. Alpha and beta signal of subject 1

(Calm)

Figure 10. Alpha and beta signal of subject 1

(Fear)

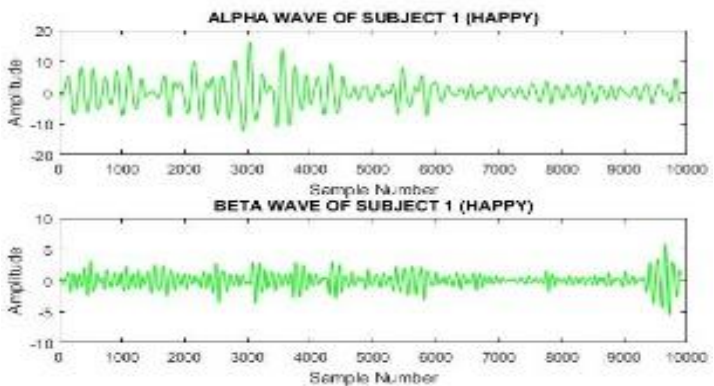

Figure 11. Alpha and beta signal of subject 1 (Happy)

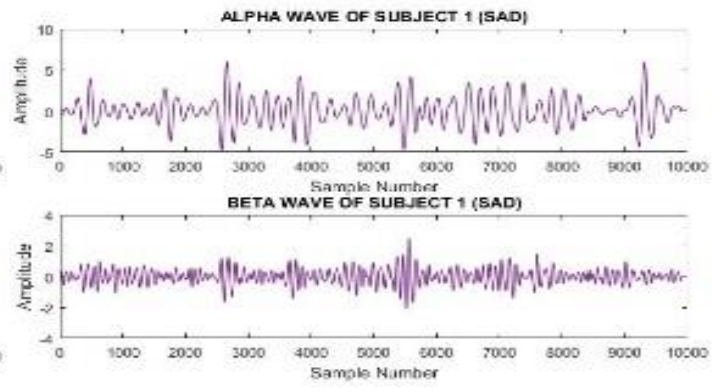

Figure 12. Alpha and beta signal of subject 1 (Sad)

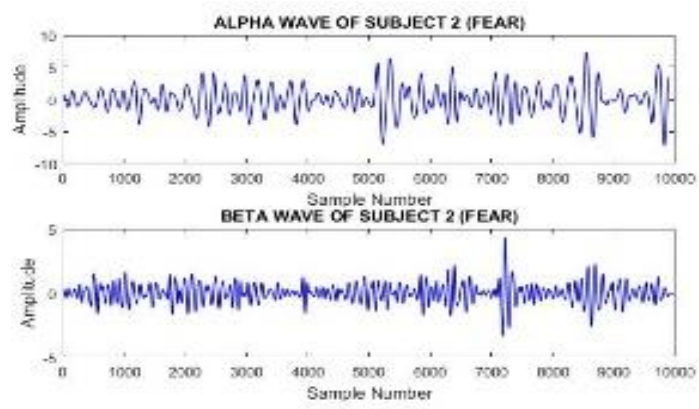

Figure 14. Alpha and beta signal of subject 2 (Fear)
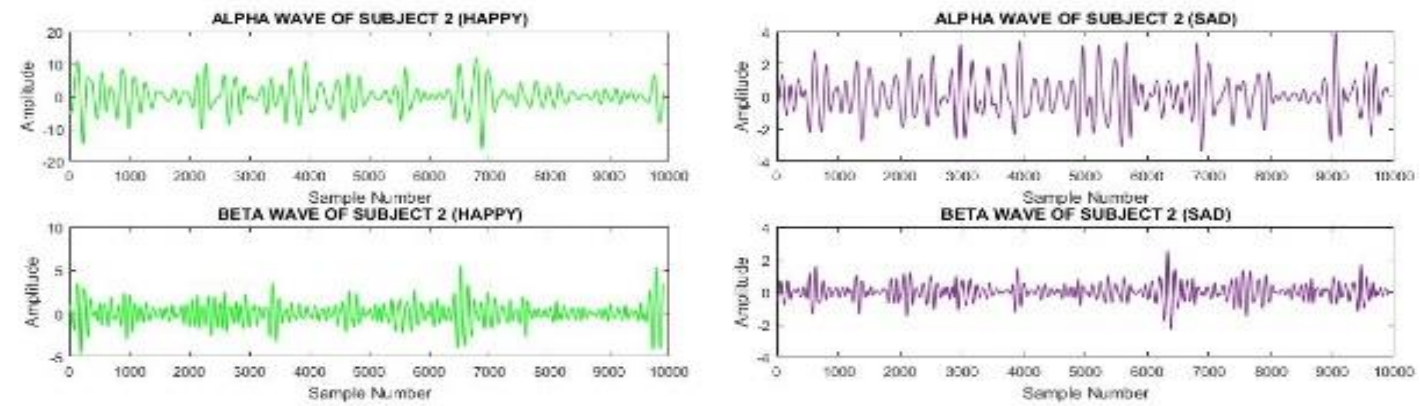

Figure 15. Alpha and beta signal of subject 2 (Happy)

Figure 16. Alpha and beta signal of subject 2 (Sad) 
The last stage of this study is classification. This is the part where all the signals will be classified according to the emotion. The data extracted from feature extraction process will be the input for this stage. In this study, MLP neural network is used by using toolbox in MATLAB R2016a. The result of the classification process for subjects 1 and 2 are shown in Figure 17 and Figure 18 respectively.

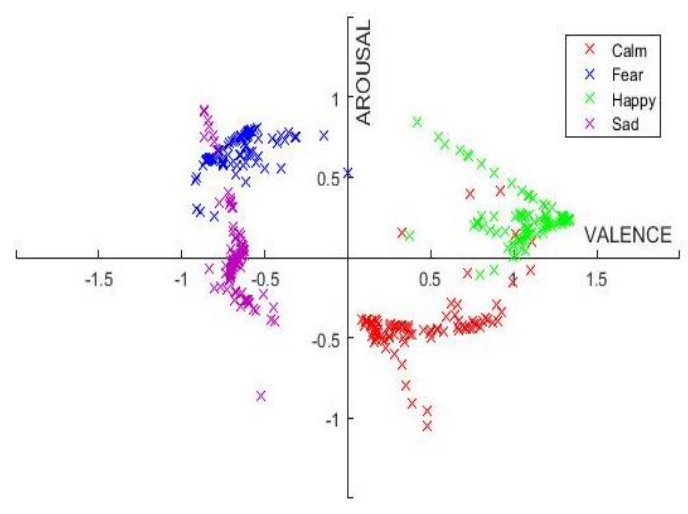

Figure 17. Emotion classification for Subject 1

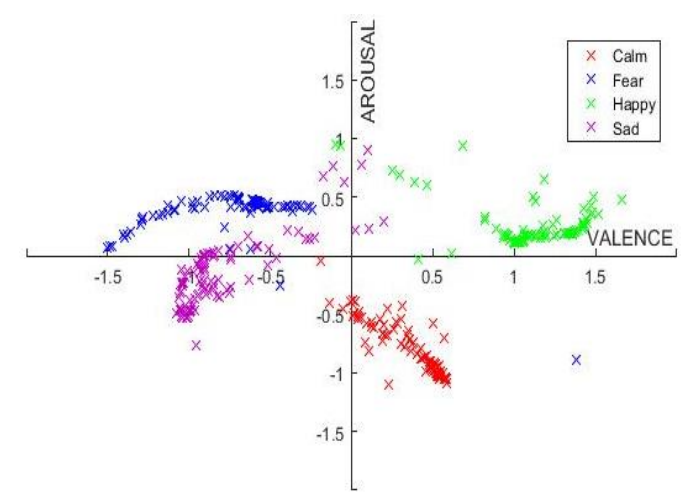

Figure 18. Emotion classification for Subject 2

Based on the result of classification process in Figure 17 until 18, majority of the signals are in the right quadrant of emotion as referring to Russell's Circumplex Model of Effect. Figures 4 show the scatter plot of the classification result after MLP neural network is applied. In each scatter plot, four emotions which are happy, fear, calm and sad are highlighted. For happy emotion, it is indicated as having positive intensity for both valence and arousal while sad emotion located at the quadrant of negative for both valence and arousal. For sad emotion, almost the entire plot indicates negative level of both valence and arousal. Meanwhile, fear emotion corresponds to positive arousal and negative valence.

However, based on the classification result, $17 \%$ of sad emotion were detected in quadrant of positive arousal. Due to psychological reason, when someone experience sad emotion, they tend to have anxiety or upset as well. Furthermore, the fear of losing someone also may contribute to high intensity of arousal as well. Table 2 shows the classification accuracies of EEG signal according to emotions for all subjects.

Table 1. Classification Accuracies of EEG Signal According to Emotions for All Subjects

\begin{tabular}{ccccc}
\hline Subjects & Calm & Fear & Happy & Sad \\
\hline Subject 1 & $85 \%$ & $92 \%$ & $92 \%$ & $90 \%$ \\
Subject 2 & $84 \%$ & $85 \%$ & $85 \%$ & $59 \%$ \\
Subject 3 & $85 \%$ & $81 \%$ & $89 \%$ & $89 \%$ \\
Subject 4 & $79 \%$ & $88 \%$ & $77 \%$ & $90 \%$ \\
Subject 5 & $82 \%$ & $89 \%$ & $82 \%$ & $82 \%$ \\
Subject 6 & $86 \%$ & $89 \%$ & $90 \%$ & $86 \%$ \\
\hline
\end{tabular}

Based on the table above, there are varying classification accuracy result as this is due to multiple emotions at the same time which causes the categorization of emotions to be mix up. However, by using the proposed method, classification accuracy up to $91 \%$ is achieved. The average classification accuracy for calm, fear, happy and sad are $83.5 \%, 87.3 \%, 85.83 \%$ and $87.6 \%$ respectively.

\section{CONCLUSION}

This study inspects the capability of EEG signal in recognizing variability of human emotion. Four steps are used to detect and recognize emotions which are data acquisition, pre-processing, feature extraction and classification of emotions. Chapter Four shows the results obtained by using the four processes mentioned before. This study proves that MLP neural network contributes well in classifying human emotions. Taking into consideration, the analysis that has been made in previous chapter, this study shows an improvised emotion recognition's system especially in differentiation four different emotions which are happy, sad, fear and calm. 


\section{ACKNOWLEDGEMENT}

I would like to extend my sincere thanks and gratitude to Department of Electrical and Computer Engineering, Kulliyyah of Engineering, IIUM and Pervasive Computing for Brain Development Research Group (PCBDG), Kulliyyah of Information and Science Technology, IIUM for assisting me in completing this study.

\section{REFERENCES}

[1] Z. Tong, K. Tong, X. Chen, and E. Al., "Emotion recognition based on photoplethysmogram and electroencephalogram,” 2018 42nd IEEE Int. Conf. Comput. Softw. Appl., pp. 402-407, 2018.

[2] C. A. Torres-valencia and M. A. Alvarez, "Comparative analysis of physiological signals and Electroencephalogram ( EEG ) for multimodal emotion recognition using generative models," 2014 XIX Symp. Image, Signal Process. Artif. Vision, Armen., pp. 1-5, 2014.

[3] Mental Health Daily, "Emotional Upheavals: Causes, Symptoms, Coping Strategies," 2015. [Online]. Available: https://mentalhealthdaily.com/2015/03/27/emotional-upheavals-causes-symptoms-coping-strategies/. [Accessed: 12-Mar-2019].

[4] G. Yang, J. Saumell, and J. Saniie, "Emotion Recognition using Deep Neural Network with Vectorized Facial Features," 2018 IEEE Int. Conf. Electro/Information Technol., pp. 318-322, 2018.

[5] P. Tarnowski et al., "Emotion recognition using facial expressions Emotion recognition using facial expressions," Procedia Comput. Sci., vol. 108, pp. 1175-1184, 2017.

[6] M. K. Ahirwal, A. Kumar, N. D. Londhe, and H. Bikrol, "Scalp Connectivity Networks for Analysis of EEG Signal during Emotional Stimulation," 2016 Int. Conf. Commun. Signal Process. (ICCSP), Melmaruvathur, pp. 592-596, 2016.

[7] J.-Y. K. Hanmoi Sim, Won-Hyung Lee, "A Study on Emotion Classification utilizing Bio-Signal ( PPG, GSR, RESP ) A Study on Emotion Classification utilizing Bio-Signal ( PPG, GSR, RESP )," IEEE Trans. Auton. Ment. Dev., vol. 7, no. 3, p. pp 162-175, 2015.

[8] C. Chen and R. E. Jack, "ScienceDirect Discovering cultural differences ( and similarities ) in facial expressions of emotion," Curr. Opin. Psychol., vol. 17, pp. 61-66, 2017.

[9] R. E. Jack, C. Blais, C. Scheepers, P. G. Schyns, and R. Caldara, "Report Cultural Confusions Show that Facial Expressions Are Not Universal," Curr. Biol., vol. 19, no. 18, pp. 1543-1548, 2009.

[10] N. Arunkumar, K. R. Kumar, and V. Venkataraman, "Entropy features for focal EEG and non focal EEG," J. Comput. Sci., pp. 2-6, 2018.

[11] U. R. Acharya, A. N. G. Peng, C. Alvin, and R. Yanti, “Application of Non-linear and Wavelet Based Identification of Epileptic EEG Signals," Int. J. Neural Syst., vol. 22, no. 2, 2012.

[12] R. F. Ahmad, A. S. Malik, N. Kamel, H. Amin, and R. Zafar, "Discriminating the Different Human Brain States with EEG Signals using Fractal Dimension : A Nonlinear Approach," 014 IEEE Int. Conf. Smart Instrumentation, Meas. Appl. (ICSIMA), Kuala Lumpur, no. November, pp. 25-27, 2014.

[13] B. Park, C. Yoon, E. Jang, and D. Kim, "Physiological Signals and Recognition of Negative Emotions," 2017 Int. Conf. Inf. Commun. Technol. Converg. (ICTC), Jeju, pp. 1074-1076, 2017.

[14] S. Poria, E. Cambria, R. Bajpai, and A. Hussain, "A review of affective computing: From unimodal analysis to multimodal fusion," Inf. Fusion, vol. 37, pp. 98-125, 2017.

[15] Engineering Medicine and Biology Society, "Biomedical Signal Processing." [Online]. Available: https://www.embs.org/about-biomedical-engineering/our-areas-of-research/biomedical-signal-processing/. [Accessed: 12-Mar-2019].

[16] H. Tjandrasa, S. Djanali, and F. X. Arunanto, "Feature Extraction Using Combination of Intrinsic Mode Functions and Power Spectrum for EEG Signal Classification," 2016 9th Int. Congr. Image Signal Process. Biomed. Eng. Informatics (CISP-BMEI), Datong, pp. 1498-1502, 2016.

[17] R. Horlings, D. Datcu, and L. Rothkrantz, "Emotion recognition using brain activity," 9th Int. Conf. Comput. Syst. Technol., pp. 1-6, 2008.

[18] D. Nie, X. Wang, L. Shi, and B. L. S. Member, "EEG-based Emotion Recognition during Watching Movies," 2011 5th Int. IEEE/EMBS Conf. Neural Eng. Cancun, pp. 667-670, 2011.

[19] M. Soleymani, S. Asghari-esfeden, M. Pantic, and Y. Fu, "Continuous emotion detection using EEG signals and facial expressions," IEEE Conf. Multimed. Expo, vol. 231287, no. 231287, pp. 3-8, 2013.

[20] H. M. Adnan, H. Yaacob, W. Abdul, and M. Othman, "Revolving Traditional EEG Device into Mobile Architecture," 2016 6th Int. Conf. Inf. Commun. Technol. Muslim World (ICT4M), Jakarta, pp. 122-127, 2016.

[21] U. R. Acharya, S. Vidya, S. Bhat, H. Adeli, and A. Adeli, "Epilepsy \& Behavior Computer-aided diagnosis of alcoholism-related EEG signals," Epilepsy Behav., vol. 41, pp. 257-263, 2014.

[22] M. Nardelli, G. Valenza, A. Greco, A. Lanata, and E. P. Scilingo, "Recognizing Emotions Induced by Affective Sounds through Heart Rate Variability," IEEE Trans. Eff. Comput., vol. 6, no. 4, pp. 385-394, 2015.

[23] N. Lim, "Cultural Differences in Emotion: Differences in Emotional Arousal Level Between The East and The West," Integr. Med. Res., vol. 5, no. 2, pp. 105-109, 2016.

[24] H. Yaacob and W. Abdul, "Classification of EEG Signals Using MLP based on Categorical and Dimensional Perceptions of Emotions," 2013 5th Int. Conf. Inf. Commun. Technol. Muslim World, 2013.

[25] J. A. Russell, “Affective Space is Bipolar,” J. Pers. Soc. Psychol., vol. 37, no. 3, pp. 345-356, 1979. 